\title{
Evaluation Des Conditions De Traitement Et De Stockage Des Plantes Médicinales Sur Les Marchés Du District d'Abidjan (Côte d'Ivoire)
}

\author{
Kouamé Kouassi Bernadin \\ Doctorant, Université Nangui Abrogoua, \\ UFR Sciences de la Nature, Ivoirien, Abidjan, Côte d’Ivoire \\ Koné Mamidou Witabouna \\ Maître de Conférence, Enseignant-Chercheur à l’Université Nangui \\ Abrogoua, UFR Sciences de la Nature, Ivoirien, Abidjan, Côte d'Ivoire \\ Chercheur associé sénior au Centre Suisse \\ de Recherches Scientifiques en Côte d'Ivoire
}

\section{doi: 10.19044/esj.2016.v13n3p396 URL:http://dx.doi.org/10.19044/esj.2016.v13n3p396}

\begin{abstract}
The present study was carried out to inventory medicinal plants storage conditions on the markets of Abidjan district. A survey was conducted from August to October 2015 in three settings of the district of Abidjan (Abobo, Adjame and Yopougon). A total of 350 sellers of medicinal plants were interrogated using semi-structured interviews. The respondents were predominantly female (98.9\%), aged between 30 and 60 years. The supply of medicinal plants in the markets was done by the wholesalers (86\%) who themselves harvested plants in different forests or savanah of Côte d'Ivoire. The majority of sellers (73\%) did not apply any measure of hygiene in storage. These people in $99.4 \%$ of case were not trained on good storage practices. Sale places were accessible to rodents and exposed to dust caused by traffic jamp. The presence of mud (9.1\%) and garbage (23.1\%) was noted in the immediate surrounding of the displays. These practices of processing and storage of medicinal plants were similar in all the visited settings. These storage conditions may constitute a hazardous risk for the consumer and justify the degradation of medicinal plants on the markets.
\end{abstract}

Keywords: Medicinal plants, Storage, herbalists, Abidjan, Côte d’Ivoire

\section{Résumé}

La présente étude a été menée en vue de faire un état des conditions de stockage des plantes médicinales sur les marchés du district d'Abidjan. Pour ce faire, une enquête a été menée d'août à octobre 2015 dans trois 
communes de la ville d’Abidjan (Abobo, Adjamé, et Yopougon). Au total, 350 commerçants de plantes médicinales ont été interrogés à l'aide d'entrevues semi-structurées. Les interviewés étaient majoritairement des femmes (98,9\%), âgés de 30 à 60 ans. Le ravitaillement en plantes médicinales sur les marchés se faisaient avec les grossistes (86\%), qui euxmêmes s’approvisionnaient dans les différentes forêts ou savanes de la Côte d’Ivoire. La majeure partie des commerçants (73\%) n’ont appliqué aucune mesure d'hygiène en matière de stockage. Ces personnes, dans 99,4\% des cas n’ont pas été formées sur les bonnes pratiques de stockage. Les lieux de vente étaient accessibles aux rongeurs et exposés aux poussières occasionnées par la circulation de véhicules. La présence de boue $(9,1 \%)$ et d’ordures $(23,1 \%)$ a été notée dans l'environnement immédiat des étalages. Ces pratiques de traitement et de stockage des plantes médicinales étaient similaires dans toutes les communes visitées. Ces conditions de conservation peuvent constituer un risque dangereux pour le consommateur et justifier l’état de dégradation des plantes médicinales sur les marchés.

Mots clés : Plantes médicinales, Stockage, Herboristes, Abidjan, Côte d'Ivoire

\section{Introduction}

Les médicaments traditionnels, et en particulier ceux à base de plantes, sont de plus en plus largement utilisés dans le monde depuis une vingtaine d'années. Malheureusement, le nombre de rapports faisant état de conséquences fâcheuses de ces produits sur la santé a aussi augmenté (OMS, 2002). Selon cette organisation, l'une des principales causes de réactions indésirables liées à l'utilisation est directement due à la mauvaise qualité des matières premières végétales et des phytomédicaments. La qualité des matières premières végétales est donc un des éléments essentiels à prendre en compte pour rendre plus sécurisante la médecine traditionnelle.

La matière première végétale peut être souvent dégradée par les microorganismes avant la récolte durant la manipulation et après une longue période de stockage (Kenneth, 1989). Des travaux menés au Ghana (AduGyamfi, 2014) et au Nigeria (Omogbai, 2013) sur des organes de plantes stockés et vendus sur les marchés, ont relevé la présence d'un large spectre de microorganismes tels que les coliformes, Escherichia coli, les Pseudomonas, Staphylococcus aureus, Aspergillus niger et Aspergillus flavus. Tous ces microorganismes sont très dangereux pour l'homme. Par ailleurs, la présence de ces microorganismes peut augmenter le taux de pourritures post-récolte des organes végétaux et entraîner ainsi un besoin fréquent de renouvellement des stocks par les herboristes. Au niveau chimique, des modifications peuvent être notées après le stockage des 
organes végétaux (Fennell et al., 2004). Par exemple, chez Vernonia colorata, Leonotis leonurus et Helichrysum cymosum, plantes utilisées dans la médicine traditionnelle, Stafford et al. (2004) ont observé une réduction ou une perte de certains composés tels que les chlorophylles, suite au stockage. Mohamed et Nezam Deldin (1985) ont également montré qu’au cours du stockage la qualité relative des polyphénols simples et des tanins solubles diminue, les flavones disparaissent en donnant des composés oxydés de couleur brune.

Des avancées significatives ont été observées dans plusieurs pays depuis la publication de la dernière stratégie mondiale concernant la médecine traditionnelle (OMS, 2012 ; 2013). Déjà en 2002, l'OMS avait fait des recommandations sur les bonnes pratiques relatives aux plantes médicinales (OMS, 2002). Ces recommandations portent principalement sur la récolte, le personnel, les installations, le transport et le conditionnement.

En Côte d'Ivoire, des travaux sur l'inventaire des plantes médicinales vendues sur les marchés ont été menés dans plusieurs communes d’Abidjan (Tra bi et al. ; 2008, Adiko et al. ; 2014, Ambé et al. ; 2015, Adon et al., 2015). Par contre, ceux portant sur les conditions de stockage et la qualité de ces plantes sont rares. Les plantes médicinales vendues sur les marchés en Côte d'Ivoire ne font pas l'objet la plupart du temps d'une inspection et d’études de qualité en vue de garantir leur sécurité et leur efficacité. Une fois sur le marché, ces plantes sont soumises aux aléas climatiques et de conservation qui peuvent affecter leurs qualités microbiologique, chimique et pharmacologique. A l'achat, le client ne sait pas en général la durée de séjour des organes végétaux à l'étalage de la vendeuse et les conditions de stockage (Kouamé, 2014).

L’objectif général de ce travail est d’évaluer les conditions de traitement et de stockage des plantes médicinales sur les marchés du district d'Abidjan en Côte d'Ivoire en comparaison avec les directives de l'OMS.

\section{Matériel et méthodes Site d'étude}

Abidjan, capitale économique de la Côte d'Ivoire, est située sur la côte atlantique entre les latitudes $4^{\circ} 10^{\prime}$ et $5^{\circ} 30^{\prime}$ Nord et les longitudes $3^{\circ} 50^{\prime}$ et $4^{\circ} 10^{\prime}$ Ouest. La ville d'Abidjan (Figure 1) est soumise à un climat attiéen chaud et humide. Les températures varient très peu toute l'année et les précipitations dépassent $1500 \mathrm{~mm}$ de pluies par an. La température moyenne mensuelle oscille entre un minimum de $24,5^{\circ} \mathrm{C}$ en août et un maximum de $29{ }^{\circ} \mathrm{C}$ en décembre, avec une moyenne annuelle de $27,2^{\circ} \mathrm{C}$. D’une superficie de $2119 \mathrm{Km}^{2}$, le district d'Abidjan regroupe une population estimée à environ 4395243 habitants (RGPH, 2014). Les marchés sont diversifiés et regorgent des produits agricoles et des plantes médicinales du pays et même 
de la sous-région. Les grands centres d'approvisionnement en plantes médicinales et alimentaires sont les marchés d’Adjamé, d’Abobo, de Yopougon et de Koumassi.

\section{Dénombrement des commerçants de plantes médicinales sur les marchés}

Trois communes, que sont Abobo, Adjamé et Yopougon, ont été sélectionnées parce qu'elles constituent les principales entrées Ouest et Nord et les points de ravitaillement des marchés du district d'Abidjan, en plantes médicinales. Un dénombrement préliminaire a permis de dénombrer tous les commerçants de plantes médicinales sur les marchés visités. Sur la base du nombre de commerçants dans chacune des communes, les marchés ayant les effectifs les plus élevés ont été retenus pour les enquêtes. Ainsi, trois marchés ont été sélectionnés dans les communes d'Abobo et d'Adjamé tandis qu'à Yopougon quatre marchés ont été visités.

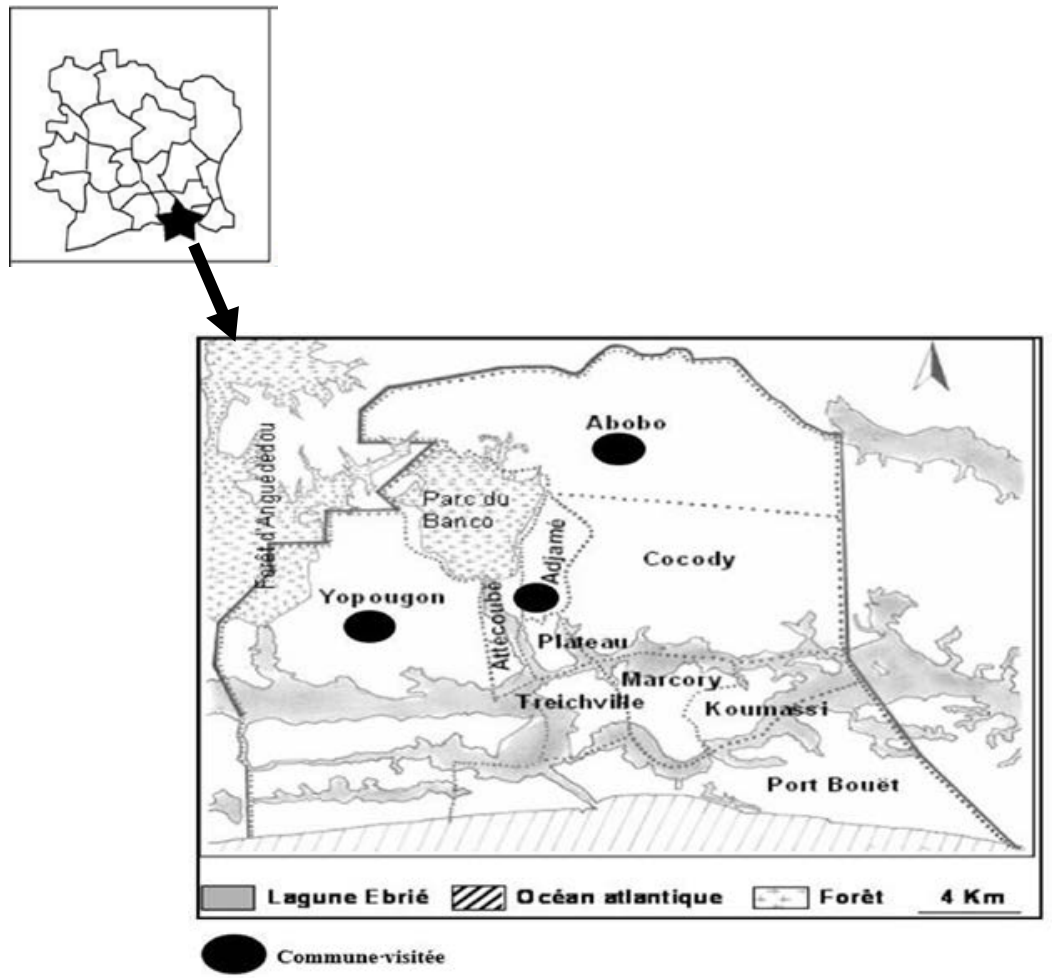

Figure 1 : Carte de la ville d’Abidjan indiquant les communes visitées (cercles noirs) 


\section{Enquêtes}

Les enquêtes ont été menées d'août à octobre 2015, à l'aide d'un questionnaire, auprès des commerçants de plantes médicinales des différentes communes (Figures 2 à 4). Le questionnaire a été établi en se référant aux recommandations de l'OMS sur les bonnes pratiques relatives aux plantes médicinales (Tableau I). Ce questionnaire a été administré à tous les commerçants présents sur les différents marchés visités durant la période des enquêtes. Les informations recueillies ont porté sur (1) le statut sociodémographique des commerçants, (2) les modes d'approvisionnements en plantes médicinales, (3) les conditions de stockage des plantes médicinales, (4) la commercialisation des plantes médicinales dans les marchés.

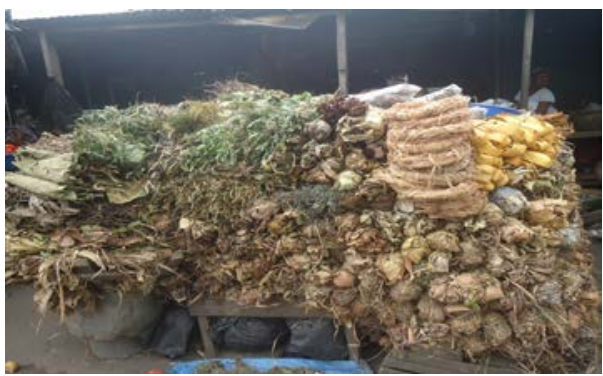

Figure 2 : Image montrant un étalage de plantes médicinales sur le marché de derrière rail à Abobo

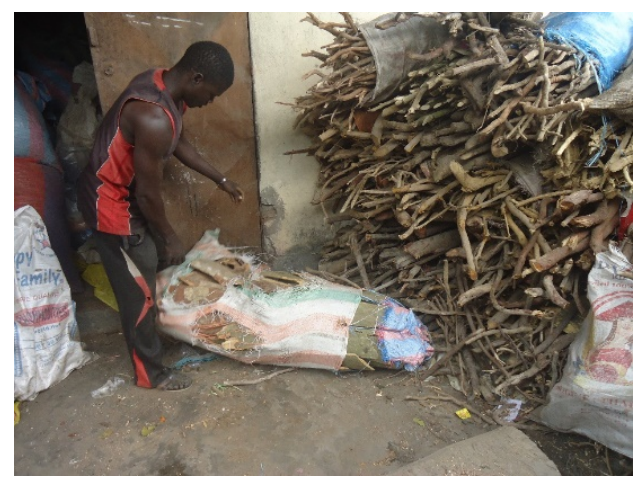

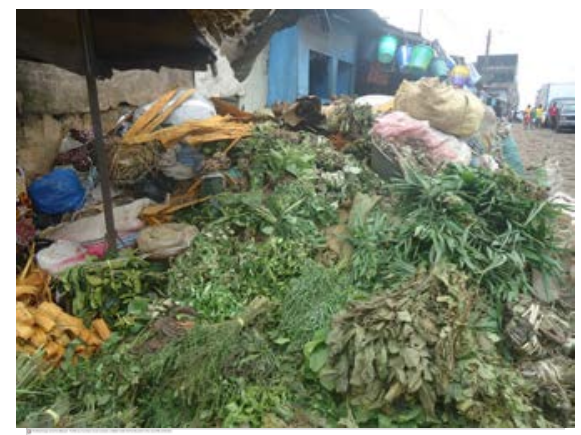

Figure 3 : Image montrant un étalage de plantes médicinales sur le marché de quartier rouge à Adjamé

Figure 4 : Image présentant des plantes médicinales stockées sur le marché de Wassakara à Yopougon 
Tableau I : Quelques recommandations de l'OMS sur les bonnes pratiques relatives aux plantes médicinales (OMS, 2002)

\begin{tabular}{|c|c|}
\hline Bonnes pratiques & Recommandations de l'OMS \\
\hline Récolte & $\begin{array}{c}\text { - Faire la récolte pendant la saison ou la période appropriée de façon à } \\
\text { assurer que les matières premières comme les produits finis seront de } \\
\text { la meilleure qualité possible. } \\
\text { - Les plantes médicinales ne doivent pas être récoltées dans des zones } \\
\text { où des quantités importantes de pesticides sont utilisées, où des dépôts } \\
\text { d’ordures et d’autres contaminants sont trouvés, ni le long des routes. } \\
\text { - Les parties de la plante qui ne sont pas utiles et les impuretés doivent } \\
\text { être enlevées. } \\
\text { - Les matières végétales médicinales brutes récoltées ne doivent pas } \\
\text { être posées directement sur le sol. } \\
\text { • Les matières récoltées seront mises dans des paniers propres, des } \\
\text { sacs en toile ou autres récipients bien aérés. } \\
\text { - Les matières végétales médicinales brutes doivent être soumises à un } \\
\text { prétraitement approprié. } \\
\text { • Les matières récoltées devront être protégées contre les insectes, } \\
\text { rongeurs, oiseaux et autres êtres nuisibles et être hors de portée du } \\
\text { bétail et des animaux domestiques. } \\
\text { • Les différentes espèces ou parties de plantes devront être } \\
\text { rassemblées séparément et transportées dans des récipients distincts. } \\
\text { • La contamination croisée doit être évitée en tout temps. }\end{array}$ \\
\hline
\end{tabular}

Tableau I : Suite

Bonnes pratiques

Personnel
Recommandations de l'OMS

- Formation théorique et pratique officielle dans le domaine des plantes.

- Les personnes présentant des plaies ouvertes, des inflammations cutanées ou des maladies de peau seront temporairement exclues du travail ou priées de porter un vêtement protecteur et des gants jusqu'à guérison complète. - Les personnes souffrant de maladies transmissibles par voie aérienne ou alimentaire, comme la dysenterie et la diarrhée, seront temporairement exclues du travail.

- Les personnes manipulant les matières végétales médicinales devront observer un niveau élevé de propreté personnelle et si nécessaire porter un vêtement protecteur et des gants, une coiffe et des chaussures.

- Les membres du personnel devront toujours se laver les mains au début des opérations de manipulation.

- Une interdiction de fumer et de manger dans les zones de manipulation des plantes médicinales.

- Les personnes manipulant les matières végétales médicinales devront éviter tout comportement qui pourrait entraîner une contamination, comme cracher, éternuer ou tousser au-dessus de matières non protégées.

- Les effets personnels tels que bijoux, montres ou autres accessoires ne devront pas être portés dans les zones de manipulation des matières végétales médicinales s’ils présentent des risques pour l'innocuité ou la qualité des produits. 
Tableau I : Suite

Bonnes pratiques

Installations

Transport
Recommandations de l'OMS

- Les installations de vente devront de préférence être situées dans un endroit exempt d'odeurs désagréables, de fumées, de poussières ou

d'autres contaminants, et se trouver en zone non inondable.

- Les bâtiments devront être de construction solide et bien entretenus.

- Tous les matériaux de construction doivent être tels qu'ils ne puissent contaminer les matières végétales médicinales par des substances indésirables.

- Toute l'installation devra disposer d'un éclairage suffisant, naturel ou artificiel.

- Une ventilation adéquate doit être assurée pour éviter toute chaleur excessive, condensation de vapeur d'eau et poussière et pour évacuer l'air vicié.

- Des installations doivent être prévues pour le stockage des déchets et des matières inutilisables avant leur évacuation.

- Les transports utilisés pour les matières végétales médicinales du site de production au site de stockage devront être nettoyés entre chaque chargement.

- Les conteneurs utilisés pour le transport, doivent être propres et le cas échéant bien ventilés afin d'éliminer l'humidité et d'empêcher la condensation.

- Les matières premières végétales doivent être transportées et stockées séparément ou d’une façon telle que leur intégrité soit préservée.

Tableau I : Suite et fin

Bonnes pratiques

Conditionnement
Recommandations de l'OMS

- Les matières végétales médicinales devront être conditionnées dans des boîtes, sacs ou autres contenants propres et secs.

- Les matériaux de conditionnement devront être non polluants, propres, secs et en bon état.

- Les matières végétales médicinales fragiles devront être conditionnées dans des récipients rigides.

- Les matériaux de conditionnement réutilisables tels que sacs de jute ou de toile devront être convenablement nettoyés (désinfectés) et séchés avant d'être réutilisés, de façon à éviter toute contamination par le contenu précédent.

- Tous les matériaux de conditionnement devront être stockés dans un lieu propre et sec, à l'abri des ravageurs et hors de portée du bétail, des animaux domestiques et autres sources de contamination.

- L'étiquette du conditionnement devra clairement indiquer le nom scientifique de la plante médicinale, la partie de la plante, le lieu d’origine de récolte, la date de récolte à l'état sauvage et le nom du récolteur, ainsi que des informations sur les quantités.

- Les matières végétales médicinales fraîches devront être stockées à basse température, de préférence à $2-8{ }^{\circ} \mathrm{C}$; les produits congelés devront être stockés à une température inférieure à $-20^{\circ} \mathrm{C}$.

- Toutes les opérations de fumigation, les fumigants utilisés et les dates des traitements devront être documentés.

- Tous les processus et modes opératoires intervenant dans la production des matières végétales médicinales, et leurs dates d'exécution, devront être documentés. 


\section{Traitement et analyse des données issues de l'enquête}

Les données de l'enquête ont été saisies en utilisant le logiciel Epidata. Les analyses statistiques ont été réalisées avec le logiciel SPSS 20. Ce logiciel a permis d'avoir les différentes proportions. L’analyse de la variance à un critère (ANOVA 1) a été utilisée pour comparer en fonction des communes, les proportions d'analphabètes, les fréquences de ravitaillement, les proportions des commerçants ne respectant pas les conditions de stockage sur les marchés, les fréquences d'écoulement des organes, les proportions des commerçants n'ayant pas reçu de formation en matière de bonnes pratiques relatives aux plantes médicinales. Pour les comparaisons multiples des valeurs moyennes de ces paramètres cités, l'ANOVA a été complétée par le test de Tukey. Ce test a permis d'identifier les pourcentages, les fréquences, les proportions qui diffèrent significativement des autres. La plus petite différence significative entre ces paramètres a été fixée à $P \leq 0,05$.

Ainsi lorsque $\mathrm{P} \geq 0,05$ on conclut qu'il n'y a pas de différence significative entre les moyennes et lorsque $\mathrm{P} \leq 0,05$ il y a une différence significative entre les moyennes.

\section{Résultats}

\section{Caractéristiques sociodémographiques des enquêtés}

Les caractéristiques sociodémographiques des commerçants interrogés sont présentées dans le tableau II. Au total, 350 commerçants de plantes médicinales ont été interviewés (Tableau III). Les personnes enquêtées sur les marchés étaient majoritairement des femmes à 98,9\%. Leur âge variait entre 30 et 60 ans, avec une proportion élevée (50,6\%) pour ceux qui ont 40 ans (Figure 5). La majeur partie des commerçants sont analphabètes avec une proportion de $62,7 \%$. Aucune différence significative n'a été oberservée par rapport au niveau d'études dans les trois communes (Figure 6). En ce qui concerne la situation matrimoniale, 93,4\% des femmes vivent en couples et ont plusieurs personnes à leur charge.

Tableau II: Caractéristiques sociodémographiques des commerçants interrogés

\begin{tabular}{ccccc}
\hline \multirow{2}{*}{ Paramètres } & \multicolumn{3}{c}{ Zone d'étude } & \multirow{2}{*}{ Moyenne } \\
\cline { 2 - 4 } & Abobo & Adjamé & Yopougon & \\
\hline Effectif & 150 & 120 & 80 & 75 \\
\hline Niveau d'étude & 119 & 83 & 22 & 42 \\
Analphabète & 31 & 37 & 58 & 0 \\
Primaire & & & 0 & 110 \\
Classe d'âge & 0 & 113 & 76 & 7 \\
[18-30[ & 140 & 7 & 4 & 42 \\
{$[30-45]$} & 10 & 38 & 13 & 75 \\
$>45$ & 73 & 82 & 67 &
\end{tabular}


Statut matrimonial Célibataire Marié

13 107
5

75
8

109

Tableau III : Répartition des enquêtes dans les différentes zones

\begin{tabular}{|c|c|c|c|c|}
\hline Ville & Communes & $\begin{array}{c}\text { Noms de marchés ou } \\
\text { quartiers }\end{array}$ & Nombres d'enquêtés & Sous-total \\
\hline \multirow{3}{*}{ Abidjan } & Abobo & $\begin{array}{c}\text { Avocatier } \\
\text { Grand marché } \\
\text { Derrière rail }\end{array}$ & $\begin{array}{l}50 \\
30 \\
70\end{array}$ & 150 \\
\hline & Adjamé & $\begin{array}{c}\text { Williamsville } \\
\text { Habitat } \\
\text { Quartier rouge }\end{array}$ & $\begin{array}{l}20 \\
20 \\
80\end{array}$ & 120 \\
\hline & Yopougon & $\begin{array}{l}\text { Wassakara } \\
\text { Siporex } \\
\text { Sicogi } \\
\text { Gesco }\end{array}$ & $\begin{array}{l}30 \\
10 \\
20 \\
20\end{array}$ & 80 \\
\hline \multicolumn{3}{|c|}{ Total } & & 350 \\
\hline
\end{tabular}

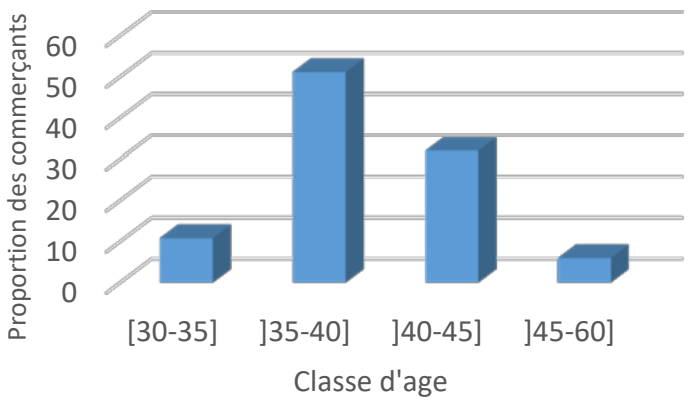

Figure 5 : Histogramme montrant les proportions des commerçants par classes d'âge

Les histogrammes portant les mêmes lettres ne montrent pas de différence significative

Figure 6 : Histogramme montrant les proportions d'analphabète en fonction des communes

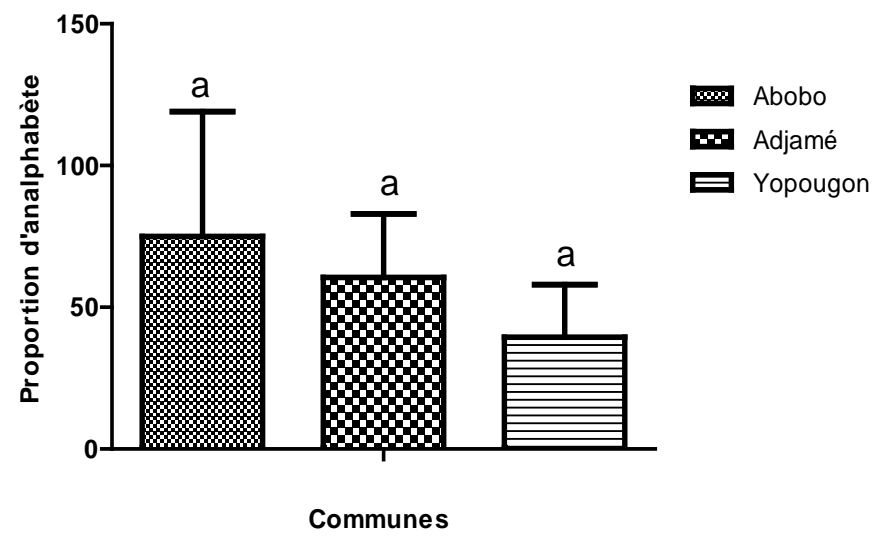




\section{Environnement de la zone de stockage}

Dans l'environnement immédiat des étalages, il a été noté la présence de boue $(9,1 \%)$ et d'ordures (23,1\%). Dans $77,1 \%$ des cas, les poussières occassionnées par la circulation de véhicules parviennent à l'étalage des vendeuses (Figure 7). Les lieux de ventes sont accessibles aux rongeurs. La présence d'usine autour des marchés n’a pas été mentionnée.

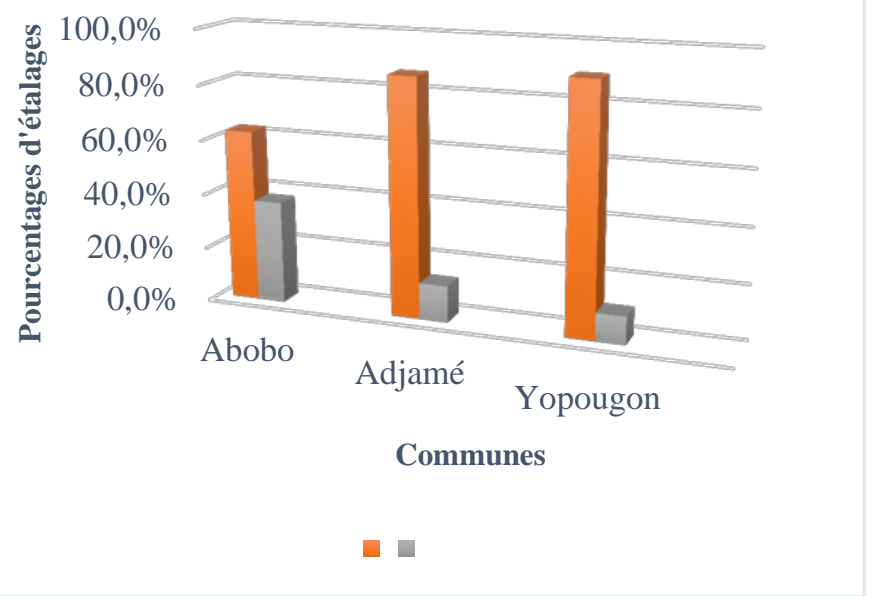

Figure 7 : Histogramme montrant les pourcentages des étalages avec présence ou absence de poussières en fonction des communes

\section{Modes d'approvisionnement en plantes sur les marchés}

La majorité des femmes (86\%) se ravitaillent avec les grossistes. Aucune différence significative n’a été observée entre les trois communes visitées en ce qui concerne le mode de ravitaillement (Figure 8). Ces grossistes n'ont aucun instrument pour contrôler l'humidité et la température au cours du transport. La date et le lieu de récolte des plantes ne sont pas en général connus des vendeuses.

Sur les 350 commerçants, 84,6\% des femmes n'ont pas de critères de qualité pour le choix de la matière première végétale livrée, seulement $15,4 \%$ de femmes ont des critères de choix.

Pour choisir leurs marchandises, les femmes considèrent quatre paramètres qui sont les caractères organoleptiques (couleur, aspect physique, odeur) (Figure 9), la présentation de la matière (organe entier, découpé, écrasé) (Figure 10), la présence d'impuretés (sables, particules de verre, moisissures ou signe de dégradation) (Figure 11) et la présence d'insectes. Pour les caractères organoleptiques, $81,8 \%$ de femmes mettent l'accent sur l'odeur comme moyen d'appréciation de l'état de dégradation de la matière, 10,9\% la couleur et 7,3\% l'aspect physique. Pour la présentation de la matière première reçue, 81,8\% reçoivent la matière brute non découpée. La majorité des femmes (92,7\%) ont 
noté la présence de sable dans les échantillons mais celle des insectes n’a pas été mentionnée par la plupart des commerçants.

Les histogrammes portant les mêmes lettres ne montrent pas de différence significative

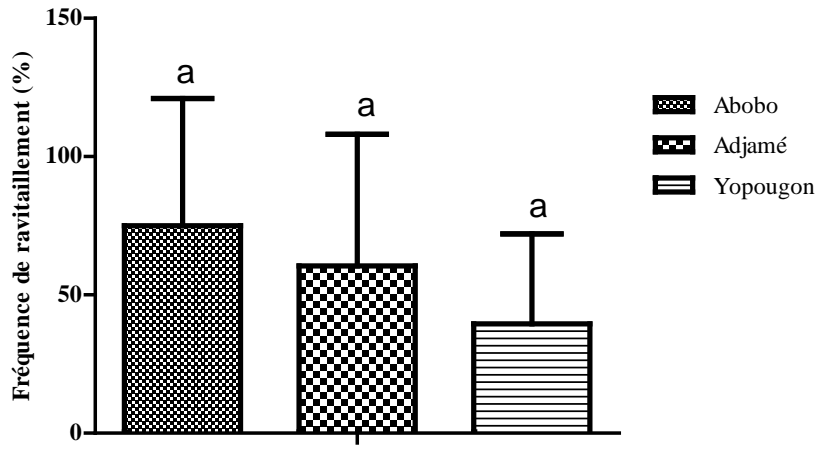

Communes

Figure 8 : Histogramme montrant les fréquences de ravitaillement des commerçants en plantes médicinales en fonction des communes

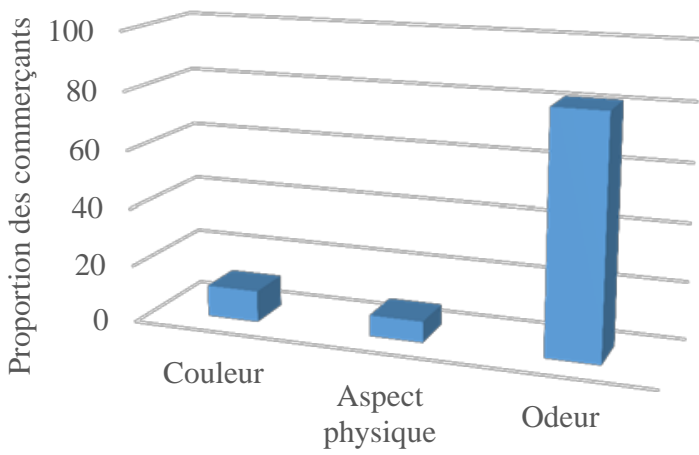

Caractères organoleptiques

Figure 9 : Histogramme montrant les proportions des commerçants considérant les caractères organoleptiques comme moyen d'appréciation de la qualité de la matière première végétale 


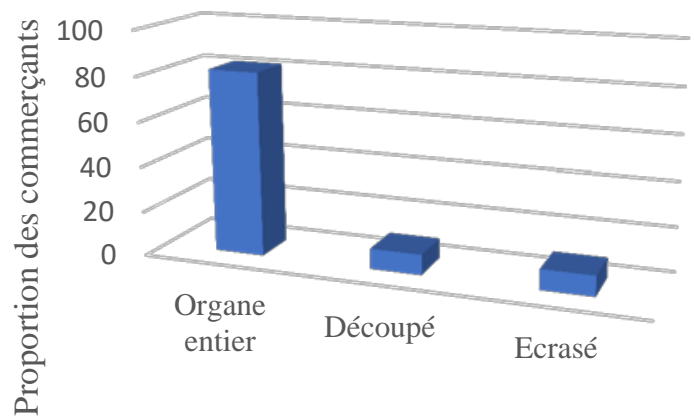

Etat de la matière

Figure 10 : Histogramme montrant les proportions des commerçants ayant reçu la matière première végétale à l’état entier, découpé ou écrasé

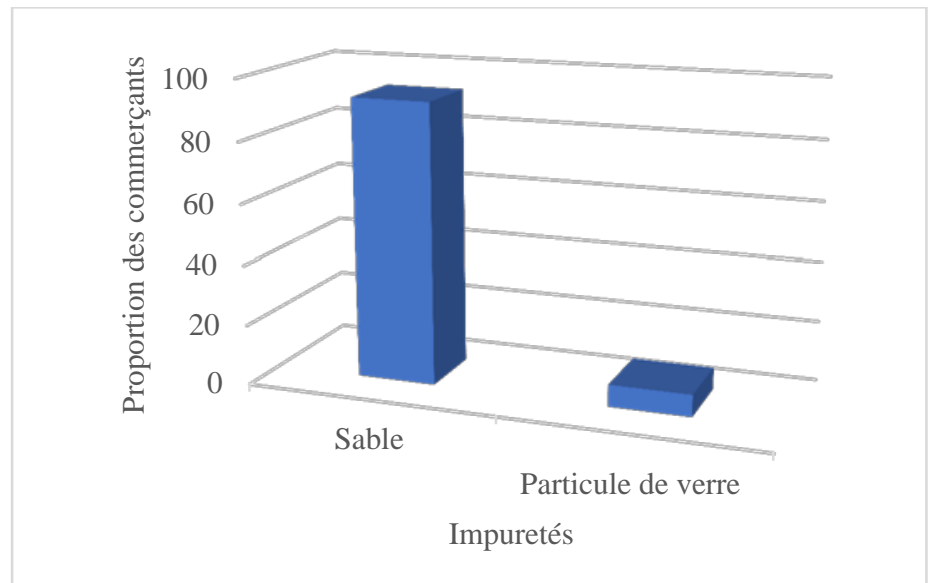

Figure 11 : Histogramme montrant les proportions des commerçants ayant déclaré avoir reçu la matière première végétale avec des impuretés

\section{Conditions de stockage des plantes sur les marchés visités}

En ce qui concerne l'aération, la protection contre la lumière et la chaleur, les stands visités étaient aérés et des parasols étaient utilisés pour la protection des organes contre la lumière et la chaleur ou comme abris pour les commerçants eux mêmes. Les rélevés des paramètres physiques tels que la température et l'humidité de l'air de la zone de stockage ne sont pas effectués.

La majeur partie des commerçants, soit $73 \%$ de femmes ne respectent aucune règle d'hygiène. Pour la proprété du matériel de stockage, il a été constacté que les commerçants (74\%) ne lavent jamais ce matériel utilisé pour couvrir les stocks. Quant à l'hygiène de la zone de stockage, seulement $46,3 \%$ des femmes fond du nettoyage. 
Une fois dans l'étalage, tous les organes de plantes sont entreposés sur les mêmes étalages sans séparation. Aucune mesure de protection contre les insectes, les animaux et les microbes n'est adoptée. Les vendeurs n'utilisent pas de produits de fumigation ou de pesticides au cours de l'entreposage. Pour couvrir les stocks après la vente, il faut noter que dans $98,9 \%$ des cas, les commerçants couvrent leurs stocks soit avec les sacs en jutes $(2,3 \%)$ ou des sachets en polyéthylène $(97,7 \%)$.

L'analyse statistique n’a montrée aucune différence significative entre les trois communes visitées, quant aux conditions de stockage. (Figure 12).

Les histogrammes portant les mêmes lettres ne montrent pas de différence significative

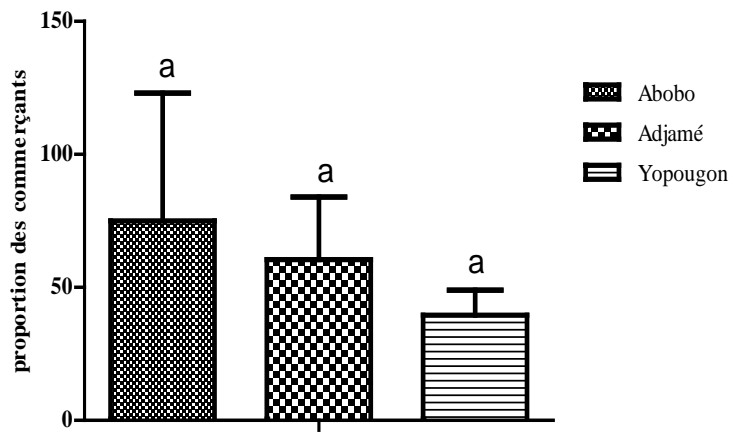

Communes

Figure 12 : Histogramme montrant les proportions des commerçants ne respectant pas les conditions de stockage sur les marchés en fonction des communes

\section{Commercialisation des plantes sur les marchés}

Les feuilles, les écorces de racines, les écorces de tiges et les poudres sont commercialisées à l'état frais ou sec par la plupart des commerçants. Par ailleurs, il faut noter la commercialisation de remèdes prêts à l'emploi tels que des décoctés et macérés à base de plantes. La quantité des organes achetés et commercialisés varie d'un à dix sacs (unité utilisée par les grossistes), avec une forte proportion pour deux sacs (44,6\%) achetés (Figure 13). On note que $99,7 \%$ des commerçants ne parviennent pas à écouler la quantité quotidienne reçue. Aucune différence significative n’a été observée entre les différentes communes en ce qui concerne l'écoulement des organes (Figure 14). Certains organes végétaux étaient encore présents dans les étalages depuis deux ans.

Dans la majorité des cas, la durée moyenne de stockage est de trois mois pour 55,1\% des 350 commerçants interrogés. Après la vente, le reste 
des organes est conservé soit dans les magasins (5,4\%), sur des tables en plein air (53,4\%), dans les sacs (40,3\%), dans les étagères (0,9\%).

En fonction du besoin ou de leur disponibilité, certains commerçants $(62,6 \%)$ travaillent avec du personnel (jeunes filles ou jeunes garçons) ayant des liens de parenté. Ce personnel a pour rôle soit le découpage $(41,1 \%)$, le lavage (12,3\%) ou même la vente des organes $(43,4 \%)$.

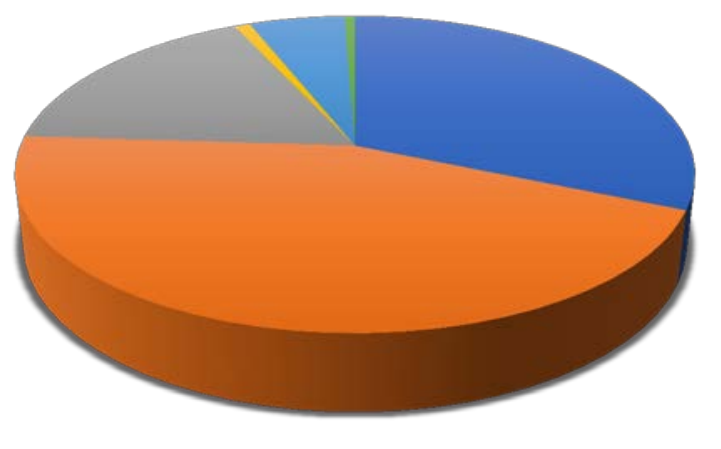

$\square$ SAC $\square 2$ SACS $\square 3$ SACS $\square 4$ SACS $\square 5$ SACS $\square 6$ SACS

Figure 13 : Diagramme montrant les quantités des plantes achetées et commercialisées dans l'ensemble des communes

Les histogrammes portant les mêmes lettres ne montrent pas de différence significative

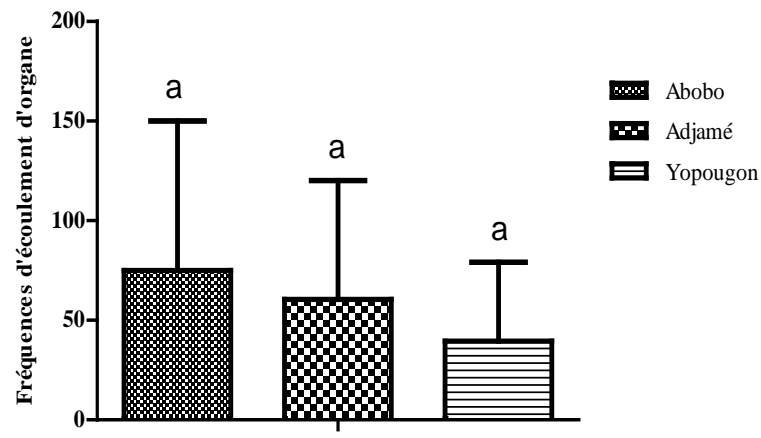

Communes

Figure 14 : Histogramme montrant les fréquences d'écoulement des organes en fonction des communes

\section{Formation des commerçants}

Un élément marquant au cours de cette enquête est que 99,4\% des femmes n'ont reçu aucune formation adéquate en matière de bonnes pratiques de stockage comme recommandé par l'OMS. Le reste de ces commerçants $(0,6 \%)$ concerne quelques tradipraticiens enregistrés par le 
programme national de la promotion de la medecine traditionnelle (PNPMT) exerçant sur ces marchés. La formation reçue a consité en générale en des seminaires sur les devoirs généraux des tradipraticiens de santé. Pour ce paramètre formation des commerçants, aucune différence significative n’a été observée entre les trois communes (Figure 15).

Les histogrammes portant les mêmes lettres ne montrent pas de différence significative

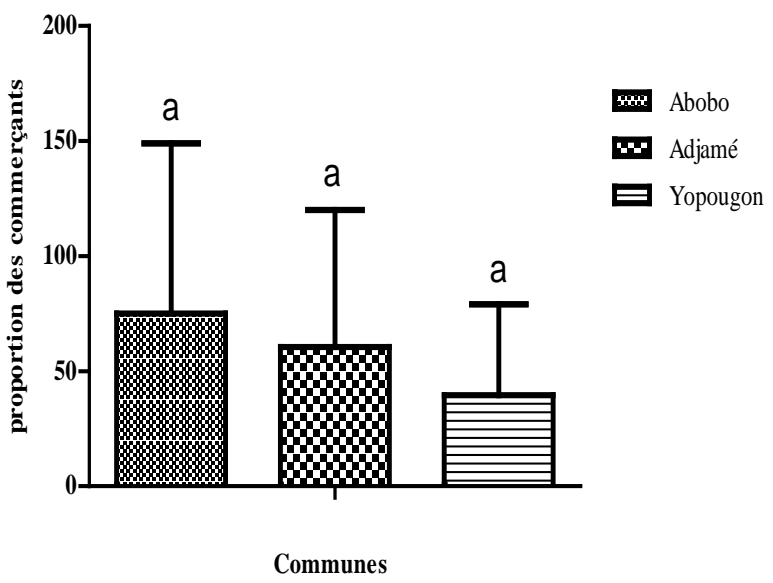

Figure 15 : Histogramme montrant les proportions de commerçants n’ayant pas reçu de formation en matière de bonnes pratiques relatives aux plantes médicinales en fonction des communes

\section{Discussion}

La présente étude a été menée en vue de faire un état des conditions de stockage des plantes médicinales sur les marchés du district d'Abidjan en comparaison des recommandations de l'OMS. Les enquêtes ont été menées sur les marchés d'Abobo, d'Adjamé et de Yopougon auprès de 350 commerçants d'août à octobre 2015.

Les résultats montrent que les enquêtés étaient majoritairement des femmes, vivant en couples et à faible revenu. Elles contribuent énormément aux charges de la famille. N'Guessan et al., (2011) et Ambe et al., (2015) ont aussi déjà fait ce même constat. Les produits forestiers non ligneux tels que les plantes médicinales jouent donc un rôle substantiel dans les moyens de subsistances des femmes. La majorité de ces commerçants sont analphabètes avec une proportion de 62,7\%. Un résultat similaire a été rapporté par Ambé et al. (2015) lors des enquêtes menées sur les mêmes marchés auprès des herboristes, $68 \%$ des herboristes interviewés étaient analphabètes. On note néanmoins une baisse de pourcentage d'analphabétisme de $5,8 \%$, ce qui est encourageant. L'âge des commerçants varie entre 30 et 60 ans, avec une forte proportion de personnes de plus de 40 ans. Les commerçants de cet âge, 
sont en général ceux qui ont plus de connaissances sur les vertus des plantes médicinales. Ce savoir est généralement acquis suite à une longue expérience accumulée et transmise d'une génération à l'autre (Klotoé et al., 2013 ; Benlamdini et al., 2014).

Les vendeuses se ravitaillent avec des grossistes qui eux-mêmes s'approvisionnent dans les différentes forêts et savanes de Côte d'Ivoire. Ils n’ont en général aucune information sur les conditions de récolte et de transport de ces plantes qu'ils vendent. Ils n'appliquent pour la plupart aucune mesure d'hygiène pendant le transport afin de préserver le bon état des organes. Ce manque de prise de précautions pourrait s'expliquer par le fait que ces personnes n'ont aucune compétence dans le domaine. Ces personnes à charge de cette activité n'ont aucune formation sur le traitement des matières premières végétales. Or selon l'OMS (2002), pour mener à bien tout ce processus une équipe chargée de la récolte familiarisée avec les bonnes techniques de récolte, de transport, de manipulation du matériel et des matières récoltées, y compris leur nettoyage, leur séchage et leur stockage, doit être mis en place. Ceci implique, un nettoyage des véhicules et des conteneurs utilisés pour le transport des matières végétales du site de production au site de stockage, une ventilation pour éliminer l'humidité et empêcher la condensation, source de prolifération d'agents biologiques de dégradation utilisés (OMS, 2002). Ces règles d'hygiène n’ont pas été observées dans ce travail au niveau de la filière plantes médicinales.

Les plantes reçues par les vendeurs ne sont pas découpées et lavées avant d'être livrées sur les marchés. La présence de sable sur les stocks a été relevée par l'ensemble des femmes. Cette présence de sable est inévitable, l'OMS (2002) préconise donc qu'après la récolte, les matières végétales médicinales brutes soient soumises à un prétraitement approprié consistant en élimination des matières indésirables et des contaminants. Ceci consiste en un lavage pour éliminer les restes de terre, un tri ou un découpage.

$\mathrm{La}$ majeur partie des commerçants ne respecte pas les règles d'hygiène en matière de stockage. Ainsi pour l'ensemble des étalages visités, on note des pratiques non conformes aux recommandation de l'OMS. Le matériel utilisé pour le découpage des organes et pour la couverture des stocks n'est pas lavé après utilisation. Les lieux de vente sont accessibles aux rongeurs et exposés aux poussières occassionnées par la circulation de véhicules. Sur les marchés visités, aucune mesure de protection des plantes médicinales contre les insectes, les animaux et les microbes n'est adoptée par les commerçants. Or plusieurs sources (FAO, 2007) rapportent que les poussières de l'environnement immédiat, les gaz d'échappement des moteurs, les mouches et autres insectes déposent des impuretés chimiques ( cuivre, plomb, fer etc.) et physiques (éclats de verre, cailloux, copeaux de métal, fil de fer, etc.) de toutes sortes sur les denrées alimentaires non 
protégés. Les plantes médicinales peuvent aussi être contaminées par ces agents chimiques et physiques. Ces composants toxiques, très persistants dans le temps, pénètrent plus ou moins rapidement et directement dans la chaîne alimentaire. Des travaux (Galsomies et al., 1999 ; Fangnon et al., 2012) ont montré que les contaminants chimiques constituent un réel danger pour l'homme. Par exemple, l'ingestion de plomb peut être à l'origine des effets neurologiques. Plusieurs auteurs dont Manisha (2014), Tatjana (2012) Nandna (2011) et Tournas (2008) ont rapporté la contamination des plantes médicinales vendues sur les marchés par des microorganismes très dangereux pour l'homme. Il ne serait donc pas surprenant que de telles contaminations ne se produisent sur les plantes vendues dans les marchés (Abobo, Adjamé et Yopougon) visités, vu les conditions de stockage.

Pour la disposition des plantes médicinales sur les étalages, les enquêtes ont revelé qu’il n’y a pas de séparation des espèces végétales, des organes, etc. Tous les échantillons commercialisés se retrouvent dans les mêmes étalages, cette manière de procéder peut occasionner des contaminations croisées des matières premières végétales. Les bonnes pratiques recommandées par l’OMS (2002) sont de rassembler séparément les différentes espèces ou parties de plantes et les transporter dans des récipients distincts afin d’éviter toute contamination croisée.

Des quantités importantes d’organes de plantes (feuilles, écorces de racines, et écorces de tige) à l'état frais ou sec sont achetées pour la commercialisation mais les stocks ne sont pas épuisés le même jour. Il se pose alors un problème de conservation des organes. Sur les marchés, on note que les commerçants réutilisent les mêmes sacs de conservation et des toiles en matière plastique pour couvrir les étalages. Ces pratiques ne sont pas conformes aux recommandations de l’OMS (2002) sur le conditionnement des plantes médicinales. L'absence des bonnes pratiques occasionnent une augmentation du taux de pourrissement post-récolte des organes végétaux. Il y a donc un besoin fréquent de renouvellement des stocks par les herboristes. Cette situation constitue une menace supplémentaire pour la biodiversité végétale.

Dans l'environnement immédiat des étalages, il a été noté la présence de boue et d'ordures. Selon FAO (2007), les lieux d'achat des matières premières et ingrédients sont nombreux et variés: champs, marchés ruraux, marchés urbains, etc. L’état et la bonne tenue des lieux d'achat sont souvent un indicateur (sans être une garantie absolue) du sérieux du commerçant. Par ailleurs, s'ils sont mal tenus, ces lieux sont susceptibles d'être des sources de contamination pour une matière première qui était de bonne qualité à l'origine, avant même son entreposage chez la vendeuse.

Selon l'OMS (2002), tous les acteurs impliqués dans la filière plantes médicinales depuis la récolte, le transport, la commercialisation et même les 
acheteurs doivent recevoir une formation adéquate en matière de bonnes pratiques de stockage, des réglements, des procédures, de la sécurité et des risques liés à la consommation de ces plantes vendues sur les marchés sans aucune réglémentation. La remarque faite au cours de cette enquête est que la quasi totalité de ces acteurs n’a reçu aucune formation.

\section{Conclusion}

Il ressort des résultats obtenus que les femmes sont les principales actrices dans la commercialisation des plantes médicinales dans le district d’Abidjan. Le mode d'approvisionnement en plantes sur les marchés, les conditions de stockages des plantes sur les marchés visités, la commercialisation des plantes sur les marchés sont loin de respecter les normes de l'OMS. La formation et la sensibilisation des acteurs aux bonnes pratiques sont donc nécessaires pour garantir la qualité de la matière première végétale. Par ailleurs, l’évaluation de la qualité microbiologique est en cours afin d’identifier les germes portés par les plantes médicinales vendues sur les marchés.

\section{References:}

1. Adiko M., Bouttier S., Okpekon T. A., Lambert S., Ake-A. L., Kablan B. J., Champy P., 2014. Phytothérapie traditionnelle des conjonctivites en milieu urbain ivoirien : enquête sur les deux marchés aux plantes médicinales d’Abidjan. Acta Botanica Gallica 161 (1), 33-45.

2. Adu-Gyamfi A., Mahami T., 2014. Effect of Drying Method and Irradiation on the microbiological quality of Moringa leaves. International Journal of Nutrition and Food Sciences 3 (2), 91-96.

3. Ambe A. S. A., Ouattara D., Tiebre M. S., Vroh bi T. A., Zirihi G. N., N'guessan K. E., 2015. Diversité des plantes médicinales utilisées dans le traitement traditionnel de la diarrhée sur les marchés d'Abidjan (Côte d'Ivoire). Journal of Animal / Plant Sciences 26 (2), 4081-4096.

4. Benlamdini N., Mohamed E., Atmane R., Lahcen Z., 2014. Étude floristique et ethnobotanique de la flore médicinale du Haut Atlas oriental (Haute Moulouya), Maroc. Journal of Applied Biosciences 78, $6771-6787$.

5. Fangnon B., Tohozin A. Y., Guedenon P., Edorh A. P., 2012. Conservation des produits agricoles et accumulation des métaux lourds dans les produits vivriers dans le département du Couffo (Benin). Journal of Applied Biosciences 57; 4168- 4176.

6. Fennell C. W., Light M. E., Sparg S. G., Stafford G. I., ELgorashi E. E., Van Staden J., 2004. Assessing African medicinal plants for 
efficacy and safety: agricultural and storage practices. Journal of Ethnopharmacology 95, 113-121.

7. Galsomiès L., Savanne D., Letrouit M. A., Ayrault S., Charre B., 1999. Retombées atmosphériques de métaux en France : estimation par dosage dans des mousses - Campagne 1996 ADEME Editions France, 187p.

8. Kenneth C., 1989. The Herb, Spice and Medicinal Plant Digest 7 (3), $1-5$.

9. Klotoé J. R., Dougnon T. V., Koudouvo K., Atègbo J-M., Loko F., Akoègninou A., Aklikokou K., Dramane K., Gbeassor M., 2013. Ethnopharmacological survey on antihemorrhagic medicinal plants in South of Benin. European Journal of Medicinal Plants 3 (1), 40-51.

10. Kouamé K. B., 2014. Etude chimique et pharmacologique dues au stockage des plantes médicinales sur trois marchés de la ville d'Abidjan (Côte d'Ivoire). Mémoire de master 2 de botanique et phytotherapie (UFR des Sciences de la Nature). Université Nangui Abrogoua (Côte d'Ivoire).

11. Manisha A., Vartika R., Sayyada K., Shanta M., 2014. Effet of microbial load on therapeutically active constituent glycyrrhizin of Glycyrrhiza glabra L. Indian Journal of Traditional Knowledge 13 (2), 319-324.

12. Mohammed N. A., Nezam Deldin A. N., 1985. A study on browing reaction in the major stages maturity of Zahidi date. Departement Date and palm Agricultural Water. Council, Baghdad, 13p.

13. N'Guessan K., Soro D., Amon A., 2011. Plantes utilisées en médecine traditionnelle dans le traitement des maladies cardiovasculaires, en pays Abbey et Krobou dans le sud de la Côte d'Ivoire. Phytothérapie 9 ; 199-208.

14. Nandna K., Rajeev K. S., Seema B., 2011. Microbiological quality assessment of some commercial herbal drugs. International Journal of Pharmaceutical Quality Assurance 3 (4) ;15-17.

15. Omogbai, Barry A., Ikenebomeh M., 2013. Microbiological characteristics and phytochemical screening of some herbal teas in Nigeria. European Scientific Journal 9 (18), 1857 -7881.

16. Organisation des Nations Unies pour l'Alimentation et l'Agriculture (FAO)., 2007. Les bonnes pratiques d'hygiène dans la préparation et la vente des aliments de rue en Afrique outils pour la formation. Rome, Italie.

17. Recensement Général de la Population et de l'Habitat (RGPH), 2014. Résultats globaux. Secrétariat Technique Permanent du Comité Technique du RGPH (Côte d'Ivoire), 3p. 
18. Stafford G. I., Jager A. K., Van Staden J., 2004. Effect of storage on the chemical composition and biological activity of several popular South African medicinal plants. Journal of Ethnopharmacology 82, 97-103.

19. Spss., 2001., SPSS for Windows release 11.5. SPSS Software Inc, Sales (USA).

20. Stratégie de l'OMS pour la médecine traditionnelle pour 2002 ; 2005. Genève, Organisation Mondiale de la Santé, (document WHO / EDM /TRM). Genève (Suisse).

21. Stratégie de l'OMS (2013) pour la médecine traditionnelle pour 2014-2023. Organisation Mondiale de la Santé. Genève (Suisse).

22. Tatjana S., Snežana P., Stanković S., and Katarina Š., 2012. Pathogenic microorganisms of medicinal herbal drugs. Archives of Biological Sciences Belgrade 64 (1), 49-58.

23. Tournas V. H., Katsoudas E. J., 2008. Microbiological quality of various medicinal herbal teas and coffee substitutes. Microbiology Insights 1, 47-55.

24. Tra B. F. H., Irié G. M., N'gaman K. C. C., Mohou C. H. B., 2008. Études de quelques plantes thérapeutiques utilisées dans le traitement de l'hypertension artérielle et du diabète : deux maladies émergentes en Côte d'Ivoire. Sciences et Nature 5 (1), 39- 48.

25. Yapi A. B., Kassi N. J., Fofie N. B. Y., Zirihi G. N., 2015. Etude ethnobotanique des Asteraceae médicinales vendues sur les marches du district autonome d'Abidjan (Côte d'Ivoire). International Journal of Biological and Chemical Science 9 (6), 2633-2647. 